\section{THE KNOWLEDGE GRADIENT FOR OPTIMAL LEARNING}

\author{
WARREN B. POWELL \\ Department of Operations Research \\ and Financial Engineering, \\ Princeton University, Princeton, \\ New Jersey
}

\section{INTRODUCTION}

There is a wide range of problems in which we need to make a decision under some sort of uncertainty, and where we have the ability to collect information in some way to reduce this uncertainty. The problem is that collecting information can be time consuming and/or expensive, so we have to do this in an intelligent way. Some examples include the following:

- We wish to find a supplier for a component who provides the lowest cost and best level of service. We know the cost, but the only way to evaluate the level of service is to try the supplier and observe actual delivery times.

- We need to find the best path through New York City to respond to certain emergencies (e.g., getting a fire truck to city hall). To evaluate the time required to traverse each path, we can try each path, or we could collect information about specific links in the network. Which paths (or links) should we measure?

- We are trying to find the best molecular compound to produce a particular result (curing cancer, storing energy, conducting electricity, etc.). There are thousands of combinations that we can try, but each test takes a day. Which compounds should we test?

- We would like to find the best price to sell a product on the internet. We can experiment with different prices and measure sales. How do we go about testing different prices?

- We have a simulation model of an operational problem (e.g., managing takeoffs and landings at Heathrow Airport). We have a number of control parameters that we can adjust to improve performance, but running a simulation takes half a day. How do we go about trying out different parameters?

- We are running a stochastic search algorithm to solve a specific optimization problem. Each iteration takes several minutes to perform a noisy function evaluation (this might be a simulation of the climate). How do we optimize this function as quickly as possible?

These are just a small number of examples of problems where we face the challenge of collecting information to make better decisions. Applications range from business decisions, science and engineering, and simulation and stochastic optimization.

The learning problems that we wish to address all have three fundamental components: a measurement decision, which determines what information we are going to collect; the information that we obtain; and an implementation decision which uses the information. We are primarily interested in sequential problems, where we can make multiple measurements and where each measurement may depend on the outcomes of previous measurements. Our goal, then, is to design a measurement policy which determines how these measurements are made.

Measurement problems come in two fundamental flavors. Off-line problems use a sequence of measurements before making a final implementation decision. These arise when we have a period of time to do research before choosing a final design. The second flavor is online problems, where we collect information each time we make an implementation decision. For example, we may

Wiley Encyclopedia of Operations Research and Management Science, edited by James J. Cochran Copyright $\odot 2010$ John Wiley \& Sons, Inc. 
want to find the best path from our new apartment in New York City to our new job. The only way to evaluate a path is to try it. The implementation decision is the choice of path to use tomorrow, but we collect information as we try the path. Off-line problems are often grouped under labels such as ranking and selection (which generally refers to choosing among a finite set of alternatives), stochastic search, and simulation optimization. Online problems are usually referred to as bandit problems. Our presentation of the knowledge gradient (KG) treats both offline and online problems in an integrated way.

Information collection problems can be approached from two perspectives: frequentist and Bayesian. In the frequentist approach, we form estimates about a truth based on information drawn from a sample (see [1-4] for excellent surveys of learning from a frequentist perspective). In this article, we focus on the Bayesian perspective, where we assume that we have a prior belief about problem parameters, along with estimates of the level of uncertainty. The Bayesian perspective assumes that there is a prior distribution of possible truths, and our goal is to design a policy that discovers this truth as quickly as possible. The frequentist perspective uses noisy observations from an unknown truth to make statistical statements about this truth; the Bayesian perspective uses a prior belief and noisy measurements to create a probability distribution to describe the truth.

In this short article, we adopt a Bayesian perspective, focusing on a concept we call the knowledge gradient, which belongs to a family of methods that guides the learning process based on the marginal value of information. The roots of this idea in the field of decision theory belong to the seminal paper by Howard [5] on the value of information. We first became aware of this idea applied as a policy for off-line ranking and selection problems from Gupta and Miescke [6]. A separate line of research that uses arose in the context of optimizing unknown functions under the general name "global optimization," with roots in the seminal paper by Kushner [7]. When these functions can only be measured with uncertainty, this field has been referred to as Bayesian global optimization, with many contributions (see Jones et $a l$. [8] for a good review). Our work in this area is based on Frazier and Powell [9], which analyzes the properties of sequential measurement policies based on the marginal value of a single measurement using a Bayesian framework with variance known; Frazier and Powell [10] provide an important generalization to problems with correlated beliefs (reviewed below). In parallel research, Chick and Branke [11] derive the marginal value of information for the important case where the variance is unknown using the name $L L(1)$ (linear loss, with measurement batches of one observation).

We provide a brief summary of different policies for collecting information. Although measurement policies may have a Bayesian or frequentist foundation, it is possible (and, we would argue, most natural) to evaluate any policy by sampling a truth from a prior distribution, and then determine how well a policy learns this assumed truth. This exercise then has to be repeated over many possible truths drawn from the prior.

The essential feature that separates learning problems from traditional stochastic optimization is that we are unsure about our uncertainty. If we make an observation, we are willing to update the probability distributions we use to describe uncertain parameters. Inserting this step of updating our beliefs after a measurement is made is typically referred to as statistical learning. When we actively make choices of what to measure, taking into account our willingness to update our beliefs, then this is optimal learning.

This article is intended to serve as a brief introduction to some of the important problem classes in optimal learning. We provide a mathematical framework for formulating and evaluating measurement policies. We review a number of heuristic policies, along with optimal policies for special problem classes. The remainder of the article focuses on the concept of the KG algorithm, which is a measurement policy that applies to a wide range of applications. 


\section{ELEMENTARY PROBLEMS}

There are two elementary learning problems that provide the foundation for our discussion. Both have a discrete set of measurements $\mathcal{X}=(1,2, \ldots, M)$, where $M$ is an integer that is "not too large," which means that we do not have any difficulty in enumerating the choices. The first problem is known in the literature as the multiarmedbandit problem, which is an online learning problem where we learn from the rewards that we receive. The second is known as the ranking and selection problem, where we have a budget of $N$ measurements to evaluate each choice, after which we have to decide which alternative appears to be best.

\section{Multiarmed Bandit Problems}

The multiarmed bandit problem is based on the story of trying to choose the best of a set of $M$ slot machines (often known as onearmed bandits). We do not know how much we will win each time we play a particular slot machine, but we have a distribution of belief, which we acknowledge may be wrong. We may think one machine has the highest expected reward, but we are willing to acknowledge that we may be wrong and another machine may also be the best. The only way we will learn is to try machines that do not appear to be the best. But while trying these machines, we may be earning lower rewards than we would earn by playing the machines that we think are better. The goal is to maximize the expected discounted sum of rewards that balance what we earn against what we learn (to improve future decisions).

Let $\mu_{x}$ be the true mean reward if we choose $x$. We do not know $\mu_{x}$, but assume that we believe that it is normally distributed with prior mean $\mu_{x}^{0}$ and variance $\left(\sigma_{x}^{0}\right)^{2}$. For convenience, we define the precision $\beta_{x}^{0}=1 /\left(\sigma_{x}^{0}\right)^{2}$. Let $W^{n}$ be the reward ("winnings") we receive in the $n$th iteration, and let $\left(\mu^{n}, \beta^{n}\right)$ be our vector of beliefs about the means and precisions for all the choices after $n$ measurements. We can write $\mu_{x}^{n}=\mathbb{E}^{n} \mu_{x}$, where $\mathbb{E}^{n}$ is the expectation given the first $n$ measurements. Let $S^{n}=\left(\mu^{n}, \beta^{n}\right)$ be our "state of knowledge" (often referred to as the belief state).
We let $x^{n}$ be the choice we make after $n$ measurements, meaning that our first choice is $x^{0}$, which is based purely on the prior. We make these measurements using a policy $\pi$, which is allowed to depend on the history of observations $W^{1}, W^{2}, \ldots, W^{n}$. Let $X^{\pi, n}\left(S^{n}\right)$ be the random variable representing the decision we make, given our state $S^{n}$, and given measurement policy $\pi$. This notation allows our policy to depend on $n$; if we wish to follow a stationary policy, we would write it as $X^{\pi, n}\left(S^{n}\right)$ (in a finite-horizon problem, the policy can depend on the number of measurements $n$, as well as on the belief state $S^{n}$ ). Our goal is to find a measurement policy $\pi$ that solves

$$
\sup _{\pi} F^{\pi}=\mathbb{E}^{\pi} \sum_{n=0}^{N} \gamma^{n} \mu_{X^{\pi, n}\left(S^{n}\right)},
$$

where $\gamma$ is a discount factor. We write the expectation $\mathbb{E}^{\pi}$ as dependent on the policy $\pi$, which reflects assumptions on how we construct the underlying probability space. We assume that an elementary outcome is a sequence of decisions of what to measure (which depend on the policy) and the results of a particular measurement. This is not the only way to construct the probability space, but it is the one that is most often used in the research community.

In the classical multiarmed bandit problem, $N=\infty$ and $\gamma<1$, but the finite horizon problem (possibly with $\gamma=1$ ) is also of interest.

\section{Ranking and Selection Problems}

Now imagine that we have a budget of $N$ measurements (or $B$ dollars to spend on measurements) after which we have to choose the best of a set of $M$ alternatives. In the bandit problem, we learn as we go, incurring rewards (or costs) as we proceed. In the ranking and selection problem, we are not concerned with how well our choices perform during the process of collecting information. Instead, we are only concerned with how well our final choice performs.

Let $\mu_{x}^{N}$ be the posterior mean of the value of alternative $x$ after $N$ measurements, which we chose using measurement policy $\pi . \mu_{x}^{N}$ is a 
random variable whose distribution depends on our measurement policy. The value of using $\pi$ can be written

$$
F^{\pi}=\mathbb{E} \max _{x}\left[\mu_{x}^{N}\right]=\mathbb{E}^{\pi} \mu_{x^{\pi}}^{N},
$$

where $x^{\pi}=\arg \max _{x} \mu_{x}^{N}$ is the optimal solution when we follow measurement policy $\pi$. The problem of finding the best policy is then given by

$$
\sup _{\pi \in \Pi} F^{\pi}
$$

We note that it is possible to write the objective as

$$
\sup _{\pi} \mathbb{E} F^{\pi}
$$

in which case we assume that the measurement policy is built into the objective function. When we write the objective this way, it means that the probability space consists of all potential measurements $W_{x}^{n}$ for all alternatives $x$ and all measurements $n$. Alternatively, we can write the objective as

$$
\sup _{\pi} \mathbb{E}^{\pi} F
$$

Written this way, it means that we have imbedded the policy into the probability space (a sample outcome consists of measurement alternatives and realizations), which means that $F$ does not explicitly depend on the policy.

\section{Notes on Objective Functions}

The formulations we have given in this section assume that we are maximizing a reward. Equivalently, we could minimize the expected opportunity cost (EOC). For the ranking and selection problem, this would be written as

$$
E O C=\mathbb{E} \max _{x} \mu_{x}-\mathbb{E} \mu_{x^{\pi}},
$$

where we are measuring the value that we could achieve if we could find the best alternative using the true means, versus the value of the alternative we did choose (but again using the true means). Keep in mind that the expectations here are over both the distribution of truths as well as the distribution over measurements. Minimizing the EOC is the same as solving

$$
\sup _{\pi} \mathbb{E} \mu_{x^{\pi}}
$$

It is also possible to show that

$$
\sup _{\pi} \sup _{\chi} \mathbb{E}^{\pi} \mu_{\chi(x)}=\sup _{\pi} \mathbb{E}^{\pi} \max _{x} \mu_{x}^{N},
$$

where $\chi$ is the policy for choosing the best alternative $x$ given what we measure, which for our problem is defined by

$$
\chi=\arg \max _{x} \mu_{x}^{N} .
$$

Equation (2) states that if we use a measurement policy to find the best alternative $x^{\pi}$ and we evaluate this choice using the true values $\mu_{x}$, we get the same answer if we evaluate our choice using the estimates $\mu_{x}^{N}$.

\section{LEARNING}

At the heart of any learning problem is not only uncertainty about the value of the choices we are making but also uncertainty about our uncertainty. Learning problems are easily posed in a Bayesian framework, where we are able to capture the uncertainty in our belief about a system. In our bandit or ranking and selection problems, $\mu_{x}$ is the true value of $x$, but we do not know this value. Instead, we assign a probability distribution that describes what we think $\mu_{x}$ is for each $x$. Before we start collecting any information, we might assume that our prior distribution of belief about $\mu_{x}$ is normally distributed with mean $\mu_{x}^{0}$ and variance $\left(\sigma_{x}^{0}\right)^{2}$. We adopt a common convention in Bayesian analysis and define the precision of our belief as $\beta_{x}^{0}=1 /\left(\sigma_{x}^{0}\right)^{2}$. Now assume that when we make an observation $W_{x}^{n}$ of choice $x$, the precision of this measurement is known and given by $\beta_{\epsilon}$. To reduce notational clutter, we assume that this is constant across $x$, but this is easily relaxed.

Assume that our current (prior) belief about choice $x$ is given by $\mu_{x}^{n}$ and $\beta_{x}^{n}$, and 
that we choose to measure alternative $x^{n}=x$. We would then observe $W_{x}^{n+1}$, which is unknown when we picked $x^{n}$. Further assume that our prior is normally distributed (i.e, $\left.\mu_{x} \sim N\left(\mu_{x}^{0}, 1 / \beta_{x}^{0}\right)\right)$, and that $W_{x}^{n+1}=\mu_{x}+\varepsilon_{x}^{n+1}$, where $\varepsilon_{x}^{n+1}$ is also normally distributed with precision $\beta_{\epsilon}$. Bayes theorem can be used to show that our updated mean and precision of the posterior belief can be computed using

$$
\begin{aligned}
& \mu_{x}^{n+1}=\frac{\beta_{x}^{n} \mu_{x}^{n}+\beta_{\epsilon} W_{x}^{n+1}}{\beta_{x}^{n}+\beta_{\epsilon}}, \\
& \beta_{x}^{n+1}=\beta_{x}^{n}+\beta_{\epsilon} .
\end{aligned}
$$

We also have the property that, if our prior belief about $\mu$ is normally distributed, then the posterior belief is normally distributed.

\section{MEASUREMENT POLICIES}

Our central challenge is designing a policy for collecting information. For off-line problems, these policies guide information collection that determines the final decision. For online problems, our policy has to strike a balance between receiving rewards (or incurring costs) and collecting information that will help future decisions.

Measurement policies can be deterministic or sequential. If we are using a deterministic policy, decisions about what to measure are made before any measurements are learned. Deterministic policies can be optimal for certain types of statistical learning problems. Our interest is in sequential problems, where the next measurement decision is made only after learning the value of the previous measurement.

We begin by showing that optimal measurement policies can be characterized using a simple dynamic programming formulation. The problem is that the dynamic program cannot be solved exactly. We then describe a series of simple heuristic policies that are often used in the research community.

\section{Optimal Sequential Policies}

Dynamic programming is widely used for sequential decision problems. For our problem, we start in a (knowledge) state $S^{n}$, then we take an action (measurement) $x^{n}$, and observe a random outcome $W_{x^{n}}^{n+1}$, which takes us to a new state according to the transition function $S^{n+1}=S^{M}\left(S^{n}, x^{n}, W^{n+1}\right)$ defined by Equations (3) and (4). Our optimal measurement $x^{n}$ can, in theory, be characterized using Bellman's equation

$V\left(S^{n}\right)=\max _{x}\left(C\left(S^{n}, x\right)+\gamma \mathbb{E}\left\{V\left(S^{n+1}\right) \mid S^{n}\right\}\right)$.

Here, $V\left(S^{n}\right)$ captures the value of being in a particular knowledge state $S^{n}$. Using the principle of dynamic programming, this is given by choosing the measurement action $x$ that maximizes the contribution earned (this might be a negative cost) from taking action $x$, plus the value of being in the knowledge state $S^{n+1}$ that results from this action, given by $V\left(S^{n+1}\right)$. Of course, $S^{n+1}$ is random given $S^{n}$ and $x$, so we have to take its expectation over the measurement $W_{x}^{n+1}$ for each alternative $x$. For off-line problems, $C\left(S^{n}, x\right)$ may be zero, since we do not receive any value until we pick the final design. For online problems, $C\left(S^{n}, x\right)$ would be the expected reward $\mu_{x}^{n}$.

So, if Equation (5) gives us the optimal measurement policy, why do we not just use this solution? The reason is that we just do not have algorithms to solve dynamic programs when the state variable is a vector of continuous parameters. There is, however, a special case that can be solved optimally without directly solving the dynamic program, which uses the concept of Gittins indices for multiarmed bandit problems. After presenting this idea, we review the most popular heuristic policies. Our presentation focuses purely on sequential policies where the choice of the $n$th measurement depends on the prior and the outcomes of $W^{1}, \ldots, W^{n}$. We then close by introducing the KG policy and illustrate the wide range of information collection problems that can be addressed using this strategy.

\section{Gittins Indices for Multiarmed Bandit Problems}

In 1974, Gittins and Jones [12] found that the multiarmed bandit problem (specifically, the infinite horizon version of the multiarmed 
bandit problem) could be solved optimally using an index policy computed using

$$
\Gamma\left(\mu_{x}^{n}, \sigma_{x}^{n}, \sigma_{\epsilon}, \gamma\right)=\mu_{x}^{n}+\Gamma\left(0, \frac{\sigma_{x}^{n}}{\sigma_{\epsilon}}, 1, \gamma\right) \sigma_{\epsilon} .
$$

In this expression, $\Gamma\left(\mu_{x}^{n}, \sigma_{x}^{n}, \sigma_{\epsilon}, \gamma\right)$ is the Gittins index for measurement $x$ for which the belief is $\mu_{x}^{n}$ and the standard deviation is $\sigma_{x}^{n} \cdot \sigma_{\epsilon}=\sqrt{1 / \beta_{\epsilon}}$ is the standard deviation of the measurement and $\gamma$ is the discount factor. The Gittins index policy, as it became known in the literature, specifies that you measure the choice $x$ with the highest value of $\Gamma\left(\mu_{x}^{n}, \sigma_{x}^{n}, \sigma_{\epsilon}, \gamma\right)$. The power of this policy is that there is one index per choice, and at no time are we dealing with multidimensional state vectors.

This leaves us with the challenge of computing $\Gamma\left(\mu_{x}^{n}, \sigma_{x}^{n}, \sigma_{\epsilon}, \gamma\right)$. Equation (6) shows us that we can compute the index as a function of a simpler index $\Gamma\left(0, \frac{\sigma_{x}^{n}}{\sigma_{\epsilon}}, 1, \gamma\right)$. This has a close parallel with the standard normal distribution (indeed, this relationship depends on normally distributed beliefs). While this relationship simplifies the problem, we still have to find $\Gamma\left(0, \frac{\sigma_{x}^{n}}{\sigma_{\epsilon}}, 1, \gamma\right)$, which requires the fairly difficult numerical solution of an integral equation.

Fortunately, there have been recent efforts to develop numerical approximations. Brezzi and Lai [13] found that

$$
\Gamma(0, s, 1, \gamma)=\sqrt{-\log \gamma} b\left(-\frac{s^{2}}{\log \gamma}\right),
$$

where the function $b(\cdot)$ has to be approximated. Chick and Gans [14] improved on the initial approximation of this function by Brezzi and Lai [13], proposing

$$
\begin{aligned}
& b(\zeta) \approx \\
& \begin{cases}\frac{\zeta}{\sqrt{2}} & \zeta \leq \frac{1}{7} \\
\exp \left(\begin{array}{cl}
-0.02645(\log \zeta)^{2} &
\end{array}\right) & \frac{1}{7}<\zeta \leq 100 \\
\sqrt{\zeta} \sqrt{\zeta \log \zeta-\log \log \zeta-\log 16 \pi} & \zeta>100 .\end{cases}
\end{aligned}
$$

The Gittins index policy is specifically designed for on-line problems, and in particular infinite horizon bandit problems. However, the structure of the policy offers some insights into the information-collection process, as we see below.

\section{Heuristic Policies}

There are a number of simple policies that can be used for collecting information. The policies reviewed below are the most popular. These can generally be adapted for on-line or off-line applications.

- Pure Exploration. Pure exploration involves picking choices at random. If there are $M$ choices, we might choose $x^{n}=x$ with probability $1 / M$ or use some other exogenously driven process. A pure exploration strategy makes little sense in an on-line application, but it can be useful (if not optimal) for off-line problems, especially for high-dimensional applications where the measurement space $\mathcal{X}$ is quite large. For off-line problems, we may use pure exploration just to collect data to fit a statistical model.

- Pure Exploitation. We make the decision that appears to be best, given what we know. Stated mathematically, a pure exploitation policy would be written as

$$
x^{n}=\arg \max _{x \in \mathcal{X}} \mu_{x}^{n} .
$$

Pure exploitation can be effective for online learning where the prior information is quite good.

- Mixed Exploration and Exploitation. Here we explore with some probability $\rho$, and exploit with probability $1-\rho$. A variation is epsilon-greedy exploration, where we explore with probability $\rho^{n}=c / n$, where $c$ is a tunable parameter.

- Boltzmann Exploration. Here we explore with probability

$$
\rho_{x}^{n}=\frac{\exp \left(\theta \mu_{x}^{n}\right)}{\sum_{x^{\prime} \in \mathcal{X}} \exp \left(\theta \mu_{x^{\prime}}^{n}\right)},
$$

where $\theta$ is a tunable parameter. $\theta=0$ produces pure exploration, while as $\theta$ increases, it approaches pure 
exploitation. This policy explores in a more intelligent way than the more classical exploration policies.

- Interval Estimation. Interval estimation is the most sophisticated of this group of heuristics. Here, we compute an index using

$$
v_{x}^{n}=\mu_{x}^{n}+z_{\alpha} \bar{\sigma}_{x}^{n} .
$$

In this policy, $z_{\alpha}$ is a tunable parameter, and $\bar{\sigma}_{x}^{n}$ is the standard deviation of our estimate $\mu_{x}^{n}$. This policy strikes a balance between the estimate $\mu_{x}^{n}$ (low values are less likely to be tested), and the uncertainty with which we know $\mu_{x}^{n}$. The policy rewards higher levels of uncertainty, but only if $\mu_{x}^{n}$ is competitive.

It is useful to compare the index policy given by interval estimation in Equation (9) with the Gittins index policy, given in Equation (6). Both policies compute an index given by the current belief plus an additional "bonus" (often referred to as the uncertainty bonus). How this bonus is computed is where the two policies differ. The Gittins policy uses a theoretically derived factor that declines to zero as the number of observations increase. This is multiplied by the standard deviation $\sigma_{\epsilon}$ of the measurement error. For interval estimation, the bonus is a constant factor (which has to be tuned) times the standard deviation $\bar{\sigma}_{x}^{n}$ of our estimate $\mu_{x}^{n}$, which then declines with the number of observations. As the number of times we measure an alternative increases, the factor $\Gamma\left(0, \frac{\sigma_{x}^{n}}{\sigma_{\epsilon}}, 1, \gamma\right)$ in Equation (6) decreases ( $\sigma_{\epsilon}$ stays the same). By contrast, with interval estimation, it is $\bar{\sigma}_{x}^{n}$ that decreases, while $z_{\alpha}$ stays the same.

\section{Policies from Simulation Optimization}

An entire body of research has developed around the problem of choosing the best set of parameters to guide a simulation. If these parameters are discrete, and if we fix the length of the simulation, this problem falls under the umbrella of ranking and selection if we are considering a finite number of alternatives. Simulation optimization introduces the additional dimension that we can choose the length of the simulation, but we may face a budget on the total computing time. This problem was first addressed under the name of "optimal computing budget allocation" (OCBA) by Chen [15]. This idea has subsequently been studied in a number of papers [16-19]. Chick and Inoue [20] introduces the $L L(B)$ strategy, which maximizes the linear loss with measurement budget $B$. He and Chick [21] introduce an OCBA procedure for optimizing the expected value of a chosen design, using the Bonferroni inequality to approximate the objective function for a single stage. A common strategy in simulation is to test different parameters using the same set of random numbers to reduce the variance of the comparisons. $\mathrm{Fu}$ et al. [22] apply the OCBA concept to measurements using common random numbers.

There is also a substantial body of literature that is often grouped under the heading of stochastic search, which addresses problems where we are searching for the best of a continuous set of parameters. For a thorough review of this field, see Spall [23]. We note, however, that this field covers numerous algorithms such as stochastic approximation methods that we would not classify as falling under the heading of optimal learning.

\section{EVALUATING POLICIES}

When faced with an array of different learning policies, we have to address the problem of evaluating policies. Using the setting of online problems, we start by observing that we generally cannot compute the expectation in Equation (1) exactly, but this equation hints at how we might evaluate a policy in practice. Let $\omega$ index both a prior $\mu(\omega)$ and a sequence of observations of $W^{1}(\omega), W^{2}(\omega), \ldots, W^{N}(\omega)$, which depend on the prior, where

$$
W_{x}^{n}(\omega)=\mu_{x}(\omega)+\varepsilon_{x}^{n}(\omega) .
$$

Recall that $X^{\pi, n}\left(S^{n}(\omega)\right)$ is our decision rule (policy) for choosing an alternative to test given our state of knowledge $S^{n}$. Given a sample path $\omega$, a sample realization of a measurement policy would be computed using 


$$
F^{\pi}(\omega)=\sum_{n=0}^{N} \gamma^{n} \mu_{X^{\pi, n}\left(S^{n}(\omega)\right)}(\omega) .
$$

Finally, we can repeat this simulation $K$ times to form a statistical estimate of the value of a policy

$$
\bar{F}^{\pi}=\frac{1}{K} \sum_{k=1}^{K} \sum_{n=0}^{N} \gamma^{n} \mu_{X^{\pi, n}\left(S^{n}\left(\omega^{k}\right)\right)}\left(\omega^{k}\right) .
$$

Now we use standard statistical tools to compare policies.

We view this method of evaluating policies as being fundamentally Bayesian, since we depend on a prior to determine the set of truths. However, the policy we are testing could be frequentist. A frequentist testing strategy might involve simulating a policy (Bayesian or frequentist) on a single function, where we start by assuming that we have no knowledge of the function (we can treat it initially as a constant), and where measurements of the function are noisy. While this strategy is not uncommon, it introduces dangers. For example, it may be possible to choose a policy that works well on a particular function. This problem can be mitigated by running the algorithm on a family of functions, but then this is comparable to generating a truth (the set of truths would be given by the family of functions).

\section{THE KNOWLEDGE GRADIENT POLICY}

A simple idea for guiding information collection is to choose to measure the alternative that provides the greatest value from a single measurement. This idea was first introduced for ranking and selection problems by Gupta and Miescke [6] as the $\left(R_{1}, \ldots, R_{1}\right)$ procedure. It has since been studied in greater depth as the knowledge gradient by Frazier and Powell [9] for problems where the measurement noise is known, and by Chick and Branke [11] under the name of $L L(1)$ (linear loss with batch size 1) for the case where the measurement noise is unknown. The idea was recently applied to on-line problems [24] for multiarmed bandit problems with both independent and correlated beliefs.
The "breakthrough," if it can be called this, with the idea of the knowledge gradient is the growing amount of empirical evidence that it appears to work well, even when competing against optimal policies such as Gittins indices for classical multiarmed bandit problems. This seems surprising, given that optimal learning problems can be formulated as dynamic programs. As a general statement, myopic policies often work poorly for dynamic problems, and the KG policy is effectively a myopic heuristic. The insight that our numerical work appears to be showing is that, while this expectation is certainly true in the context of dynamic problems that arise in the management of physical resources, it does not appear to hold true in the context of learning problems.

\section{The Knowledge Gradient for Off-Line Learning}

As before, we assume that all beliefs are normally distributed with parameters captured by the state $S^{n}=\left(\mu_{x}^{n}, \beta_{x}^{n}\right)_{x \in \mathcal{X}}$. Given $S^{n}$, which means, given our beliefs about each choice $\mu_{x}^{n}$, the value of our current state of knowledge is given by

$$
V^{n}\left(S^{n}\right)=\max _{x^{\prime} \in \mathcal{X}} \mu_{x^{\prime}}^{n}
$$

Now assume that we choose to measure $x^{n}=x$. This means that we get to observe $W_{x}^{n+1}$ and update our belief about $\mu_{x}$. We write this updating process using our transition equation $S^{n+1}(x)=S^{M}\left(S^{n}, x^{n}\right.$, $\left.W^{n+1}\right)$. This means applying the Bayesian updating Equations (3) and (4), but only for choice $x$. With this new information, the value of our new state of knowledge $S^{n+1}$ would be given by

$$
V^{n+1}\left(S^{n+1}(x)\right)=\max _{x^{\prime} \in \mathcal{X}} \mu_{x^{\prime}}^{n+1} .
$$

At iteration $n$, however, the observation $W^{n+1}$ is a random variable, which means that $V^{n+1}\left(S^{n+1}(x)\right)$ is a random variable. We can compute the expected value of measuring $x$ as

$v_{x}^{\mathrm{KG}, n}=\mathbb{E}\left[V^{n+1}\left(S^{n+1}(x)\right)-V^{n}\left(S^{n}\right) \mid S^{n}\right]$.

We refer to $v_{x}^{\mathrm{KG}, n}$ as the $K G$ since it is the marginal value of information from 
Table 1. Calculations Illustrating the Knowledge Gradient Index

\begin{tabular}{lccccccc}
\hline Measurement & $\mu$ & $\bar{\sigma}$ & $\beta$ & $\tilde{\sigma}$ & $\zeta$ & $f(\zeta)$ & $v_{x}^{\mathrm{KG}, n}$ \\
\hline 1 & 20.0 & 18.00 & 0.0031 & 17.999 & -0.444 & 0.215 & 3.878 \\
2 & 22.0 & 12.00 & 0.0069 & 11.998 & -0.500 & 0.198 & 2.373 \\
3 & 24.0 & 25.00 & 0.0016 & 24.999 & -0.160 & 0.324 & 8.101 \\
4 & 26.0 & 12.00 & 0.0069 & 11.998 & -0.167 & 0.321 & 3.853 \\
5 & 28.0 & 16.00 & 0.0039 & 15.999 & -0.125 & 0.340 & 5.432 \\
\hline
\end{tabular}

measuring $x$. Note that the KG policy not only captures what we learn about the mean of a choice but also the change in the precision of our belief. We write the KG policy as choosing to measure the alternative $x$ which has the highest marginal value of information. We write this policy simply as

$$
X^{\mathrm{KG}, n}=\arg \max _{x \in \mathcal{X}} v_{x}^{\mathrm{KG}, n} .
$$

We start by computing the variance of the change in our estimate of $\mu_{x}$ given our state of knowledge $S^{n}$, given by

$$
\tilde{\sigma}_{x}^{2, n}=\operatorname{Var}\left[\mu_{x}^{n+1}-\mu_{x}^{n} \mid S^{n}\right] .
$$

It is fairly straightforward to show that

$$
\begin{aligned}
\tilde{\sigma}_{x}^{2, n} & =\bar{\sigma}_{x}^{2, n}-\bar{\sigma}_{x}^{2, n+1}, \\
& =\frac{\left(\bar{\sigma}_{x}^{2, n}\right)}{1+\sigma_{\epsilon}^{2} / \bar{\sigma}_{x}^{2, n}} .
\end{aligned}
$$

We then compute the distance between our current estimate of the value of $x$, and the best of the rest, normalized by the number of standard deviations in the measurement of the change, given by

$$
\zeta_{x}^{n}=-\left|\frac{\mu_{x}^{n}-\max _{x^{\prime} \neq x} \mu_{x^{\prime}}^{n}}{\tilde{\sigma}_{x}^{n}}\right| .
$$

Next, we use a standard formula for $\mathbb{E} \max \{0, Z+\zeta\}$, where $Z$ is the standard normal deviate. This formula is given by

$$
f(\zeta)=\zeta \Phi(\zeta)+\phi(\zeta)
$$

where $\Phi(\zeta)$ and $\phi(\zeta)$ are respectively the cumulative standard normal distribution and the standard normal density. Finally, the $\mathrm{KG}$ is given by

$$
v_{x}^{\mathrm{KG}, n}=\tilde{\sigma}_{x}^{n} f\left(\zeta_{x}^{n}\right) .
$$

For a more detailed development of these equations, see [9]. Table 1 provides an illustration of the calculations for a simple problem with five alternatives.

\section{On-Line Learning}

The ranking and selection problem has a long history that has evolved completely independently of the multiarmed bandit problem. However, we can take the basic idea of the KG policy and apply it to the multiarmed bandit problem. We have to find the expected value of a single measurement. For on-line problems, we approach this by assuming that we are going to do a single step from which we can learn and update our beliefs about the problem. After that, we continue making decisions holding our state of knowledge constant.

If we have an on-line learning problem where we have only two trials, this means we can learn from the first trial and use it in the second trial. The value of measuring $x$ would simply be $v_{x}^{\mathrm{KG}, n}$, which measures the improvement in our ability to solve the problem in the second trial.

Now imagine that we have $N$ trials, where we have already made $n-1$ measurements and are thinking about what we should do for the $n$th measurement. Since this is an on-line problem, the expected reward we will receive by measuring $x$ is $\mu_{x}^{n}$. In addition, we get the benefit of using this information $N-n$ more times in later decisions. Using this reasoning, we can find the on-line knowledge gradient (OLKG) using

$$
v_{x}^{\mathrm{OLKG}, n}=\mu_{x}^{n}+(N-n) v_{x}^{\mathrm{KG}, n} .
$$

If we have an infinite horizon problem (as with the standard multiarmed bandit problem) with discount factor $\gamma$, the OLKG policy would be 


$$
v_{x}^{\mathrm{OLKG}, n}=\mu_{x}^{n}+\frac{\gamma}{1-\gamma} v_{x}^{\mathrm{KG}, n}
$$

Again note the comparisons against the Gittins index policy and the interval estimation policy, both of which use the expected reward from a single measurement, and a term that in some way captures the value of information on future decisions (although the OLKG policy is the only one of these policies where this is explicit). Unlike these other two policies, on-line KG is not a true index policy, since $v_{x}^{\mathrm{KG}, n}$ depends on a calculation that requires information from all the other choices. The Gittins and IE index policies compute an index that is based purely on parameters from a particular choice.

This idea is developed with much greater rigor in Ryzhov et al. [24], which also reports on comparisons between the OLKG policy and other policies. It was found that the $\mathrm{KG}$ policy actually outperforms Gittins indices even when applied to multiarmed bandit problems (where Gittins is known to be optimal) when we use the approximation in Equation (8). KG also outperforms pure exploitation (i.e., for on-line problems), but it slightly underperforms interval estimation when the parameter $z_{\alpha}$ is carefully tuned. However, it was also found that IE is sensitive to this tuning, and even slight deviations from the best value of $z_{\alpha}$ can produce results that underperform KG.

\section{The S-Curve Problem}

An issue with the $\mathrm{KG}$ is that the value of information can be nonconcave. It is not hard to create problems where a single measurement adds very little value, since the noise in the observation is too high to change a decision. We can easily find the value of information by computing the $\mathrm{KG}$ where the precision of a measurement $x$ is given by $n_{x} \beta_{\epsilon}$ rather than just $\beta_{\epsilon}$. Think of it as making a single measurement with precision $n_{x} \beta_{\epsilon}$ instead of $\beta_{\epsilon}$. An example of a curve where the value of information is nonconcave is given in Fig. 1.

The issue of the nonconcavity of information is well known. Howard [5] demonstrates the nonconcavity of information in the context of an auction problem. Radner and Stiglitz [25] discusses the problem in considerably greater depth, giving general conditions under which the value of information is not concave. This research has been extended more recently by Chade and Schlee [26] and Delara and Gilotte [27] to more general settings.

The issue is discussed in Frazier and Powell [28] in the context of sequential sampling. This paper proposes a modification of the KG policy, which is dubbed $\mathrm{KG}(*)$. In this policy, we find the value of $n_{x}$, which maximizes the average value of information, as depicted in Fig. 1. We note that in a budget-constrained setting, we may not have the budget to do $n_{x}$ measurements, in which case we have to consider the value of a smaller number of measurements. Further, we may not wish to use our entire budget on a single alternative, which may eliminate alternatives that require a large number of measurements to evaluate.

\section{Correlated Beliefs}

The KG policy is able to handle the very important case of correlated beliefs. This arises when measuring $x$ may tell us something about $x^{\prime}$. For example, imagine that we are testing the set of features in a product, or we want to identify the best set of drugs to put into a drug treatment, or we want to evaluate the best set of energy saving technologies in a house. These are all instances of subset (or portfolio) selection problems, where a choice $x$ might be given by

$$
x=(0,1,1,0,1,0,0,0) .
$$

Now consider a choice $x^{\prime}$ given by

$$
x^{\prime}=(0,1,1,0,0,1,0,0) .
$$

$x$ and $x^{\prime}$ share two common members, so it might be reasonable to expect that, if $x$ performs better than expected, that $x^{\prime}$ will perform better than expected. Let $\Sigma^{0}$ be our initial estimate of the covariance in our belief between different alternatives, where $\Sigma^{0}$ has $|\mathcal{X}|$ rows and columns. Also, let $B^{n}=\left(\Sigma^{n}\right)^{-1}$ be the inverse of the covariance matrix (think of this as the matrix version of our precision). Assume that we measure $x^{n}=x$, observing $W_{x}^{n+1}$. Recalling that $\mu^{n}$ is our vector of 


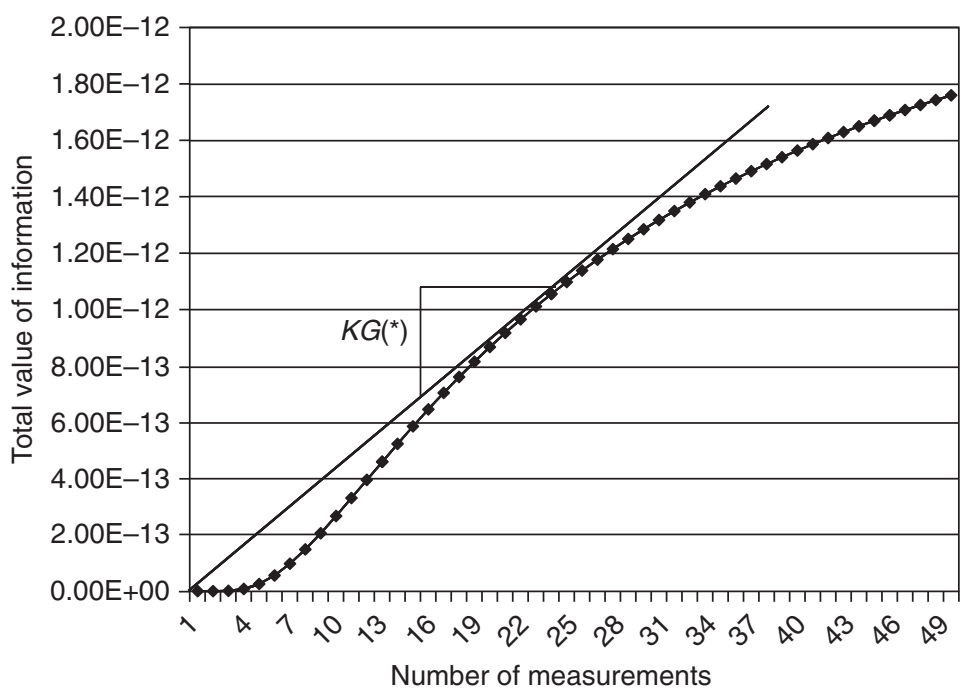

Figure 1. Illustration of the nonconcavity of information, and the maximum average value that would be used by the $\mathrm{KG}(*)$ algorithm. beliefs, let $\mu^{n+1}(x)$ be the updated vector of beliefs assuming we measure $x=x^{n}$, and let $\Sigma^{n+1}(x)$ be the updated matrix of covariances. The Bayesian formulas for updating means and covariances, in the presence of correlated beliefs, are given by

$$
\begin{aligned}
& \mu^{n+1}(x)=\Sigma^{n+1}(x)\left(B^{n} \mu^{n}+\beta_{\epsilon} W_{x}^{n+1} e_{x}\right), \\
& B^{n+1}(x)=B^{n}+\beta_{\epsilon} e_{x}\left(e_{x}\right)^{T},
\end{aligned}
$$

where $e_{x}$ is a column vector of $0 \mathrm{~s}$ with a 1 in the element corresponding to alternative $x$.

For our purposes, there is a more convenient way of expressing the updating of the vector $\mu^{n}$. First, let $\tilde{\Sigma}^{n}(x)$ be the change in the covariance matrix for $\mu^{n}$ due to measuring alternative $x$, just as $\tilde{\sigma}_{x}^{2, n}$ was the change in the variance of $\mu_{x}^{n}$ due to measuring $x$. This is given by

$$
\begin{aligned}
\tilde{\Sigma}^{n}(x) & =\Sigma^{n}-\Sigma^{n+1}(x) \\
& =\frac{\Sigma^{n} e_{x}\left(e_{x}\right)^{T}}{\sqrt{\Sigma_{x x}^{n}+\lambda^{W}}} .
\end{aligned}
$$

Next let $\tilde{\sigma}^{n}(x)$ be the column vector of $\tilde{\Sigma}^{n}(x)$ corresponding to alternative $x$, given by

$$
\tilde{\sigma}^{n}(x):=\tilde{\Sigma}^{n}(x) e_{x} .
$$

Also, let $\tilde{\sigma}_{i}^{n}(x)=\left(e_{i}\right)^{T} \tilde{\sigma}^{n}(x)$ be the $i$ th component of the vector $\tilde{\sigma}^{n}(x)$.
Let $\operatorname{Var}^{n}[\cdot]=\operatorname{Var}\left[\cdot \mid S^{n}\right]$ be the variance given all the measurements up through iteration $n$. We observe that we can write

$$
\begin{aligned}
\operatorname{Var}^{n}\left[W_{x}^{n+1}-\mu_{x}^{n}\right] & =\operatorname{Var}^{n}\left[W_{x}^{n+1}\right] \\
& =\operatorname{Var}^{n}\left[\mu_{x}+\varepsilon_{x}^{n+1}\right] \\
& =\Sigma_{x x}^{n}+\sigma_{\epsilon}^{2},
\end{aligned}
$$

where the last step follows from the independence between the belief about the variance of $\mu_{x}$ conditioned on $S^{n}$, and the noise in the $n+1$ st measurement $\varepsilon_{x}^{n+1}$. Now let

$$
Z^{n+1}:=\left(W^{n+1}-\mu^{n}\right) / \sqrt{\operatorname{Var}^{n}\left[W^{n+1}-\mu^{n}\right]},
$$

where $\operatorname{Var}^{n}\left[W^{n+1}-\mu^{n}\right]=\Sigma_{x x}^{n}+\sigma_{\epsilon}^{2}$. It is straightforward to show that $Z^{n+1}$ is normally distributed with mean 0 and variance 1. We can now write the updating equation using

$$
\mu^{n+1}=\mu^{n}+\tilde{\sigma}^{n}\left(x^{n}\right) Z^{n+1} .
$$

Also, it is easy to see that $\operatorname{Var} Z^{n+1}=1$ (since we constructed it that way).

The KG policy for correlated measurements is computed using 
Figure 2. Regions of $z$ over which different choices dominate. Choice 2 is always dominated.

$$
\begin{aligned}
v_{x}^{\mathrm{KG}} & =\mathbb{E}\left[\max _{i} \mu_{i}^{n+1} \mid S^{n}=s, x^{n}=x\right] \\
& =\mathbb{E}\left[\max _{i} \mu_{i}^{n}+\tilde{\sigma}_{i}^{n}\left(x^{n}\right) Z^{n+1} \mid S^{n}, x^{n}=x\right],
\end{aligned}
$$

where $Z$ is a scalar random normal variate.

Our challenge now is computing this expectation, which is much harder for correlated beliefs, but the computation is very manageable. We start by defining

$$
\begin{aligned}
& h\left(\mu^{n}, \tilde{\sigma}^{n}(x)\right) \\
& \quad=\mathbb{E}\left[\max _{i} \mu_{i}^{n}+\tilde{\sigma}_{i}^{n}\left(x^{n}\right) Z^{n+1} \mid S^{n}, x^{n}=x\right] .
\end{aligned}
$$

Substituting Equation (18) into Equation (17) gives us

$$
X^{\mathrm{KG}}(s)=\arg \max _{x} h\left(\mu^{n}, \tilde{\sigma}^{n}(x)\right) .
$$

Let $\quad h(a, b)=\mathbb{E}\left(\max _{i} a_{i}+b_{i} Z\right), \quad$ where $a_{i}=\mu_{i}^{n}, \quad b_{i}=\tilde{\sigma}_{i}^{n}\left(x^{n}\right)$ and $Z$ is our standard normal variate. $a$ and $b$ are $M$ (or $|\mathcal{X}|)$-dimensional vectors of means and $\tilde{\sigma}$ 's. Now sort the elements of the vector $b$ so that $b_{1} \leq b_{2} \leq \ldots$, giving us a sequence of lines with increasing slopes. Looking at the lines $a_{i}+b_{i} z$ and $a_{i+1}+b_{i+1} z$, we find they intersect at

$$
z=c_{i}=\frac{a_{i}-a_{i+1}}{b_{i+1}-b_{i}} .
$$

Assume that $b_{i+1}>b_{i}$. If $c_{i-1}<c_{i}<c_{i+1}$, then there should be a range for $z$ over which a

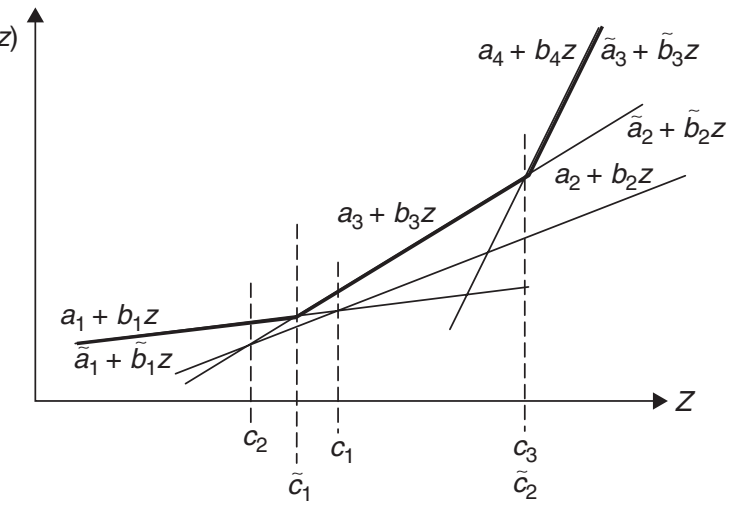

particular choice dominates, as depicted in Fig. 2. It is possible that a choice is always dominated, which will happen if $c_{i+1}<c_{i}$ (see choice 2 in the Fig. 2). If this happens, we simply drop the choice from the set, producing a new set of choices with coefficients $\tilde{a}_{i}, \tilde{b}_{i}$ and intersections $\tilde{c}_{i}$, which satisfy $c_{i-1}<c_{i}<c_{i+1}$.

Once we have sorted the slopes and dropped dominated alternatives, we can compute Equation (17) using

$$
h(a, b)=\sum_{i=1}^{M}\left(b_{i+1}-b_{i}\right) f\left(-\left|c_{i}\right|\right),
$$

where, as before, $f(z)=z \Phi(z)+\phi(z)$. Note that the summation over alternatives has to be adjusted to skip any choices $i$ that were found to be dominated. For a more detailed presentation, see Frazier et al. [10].

The ability to handle correlated beliefs is a major generalization, and one which is not possible with the other heuristic search rules or Gittins indices. There are numerous practical problems where these correlations might be expected to arise. For example, if we are measuring a continuous function, we would expect our beliefs about $x$ and $x^{\prime}$ to be correlated in proportion to the distance between $x$ and $x^{\prime}$. Correlated beliefs make it possible to tackle problems where the measurement budget is potentially much smaller than the number of potential measurements.

There is a limited but growing literature on bandit problems (online learning problems) where there is structure among the alternatives. Berry and Fristedt [29], Chapter 2 consider the case of correlated 
beliefs, but do not provide a method to solve it. Pandey et al. [30] introduces the notion of dependent arms where alternatives can be grouped into clusters of arms with similar behavior. The choice is then reduced to one of choosing among clusters. Presman and Sonin [31] introduces the idea of bandit problems with hidden state variables, which creates dependencies among different alternatives. There is a small but growing literature on problems where correlations are introduced by assuming an underlying functional form for the belief [32].

Finally, there are a number of papers that deal with the important special case of continuous functions. Ginebra and Clayton [33] studies "response surface bandits" where the measurements are continuous; they introduce a simple heuristic (basically an uncertainty bonus similar to Gittins indices) for learning a surface. Agrawal [34] considers learning a multidimensional surface, but this is accomplished by sampling the function at equally spaced points, and then combining the results to produce an estimate of the function using kernel regression [35]. Kleinberg [35] improves on this by sampling over evenly spaced intervals, but then applying a standard bandit algorithm to the resulting finite set of points (there is no evidence that correlations are being used in the choice of what to measure).

\section{Other Applications of the Knowledge Gradient}

Now that we have shown that we can use the $\mathrm{KG}$ concept for on-line and off-line problems, as well as the important class of correlated beliefs, it is natural to ask the following question: what is the scope of applications of the $\mathrm{KG}$ for guiding information collection? We have only begun to explore the potential range of applications, but some examples include the following:

- Learning on Graphs. Consider the problem of making measurements so that we can better learn the costs on an uncertain graph. For example, we need to find the best path for an emergency response vehicle, and we have to send people to measure the costs on specific links of the network to produce the best solution to our shortest path problem. Ryzhov and Powell [36] shows that we can identify the best link to evaluate using a method that is very similar to the KG for independent beliefs.

- Drug Discovery. Negoescu et al. [37] consider the problem of finding the best molecular compound, through intelligent sequencing of laboratory experiments. The research used a linear regression model to capture beliefs, reducing a problem with 87,000 possible compounds to one involving only 200 regression parameters. The KG policy was able to identify a compound very close to the best in under 100 experiments.

- Subset Selection. Ryzhov and Powell [38] shows how the KG can be used to guide the selection of the best set of energy-saving technologies to implement in a building, recognizing that different technologies interact in different ways for a particular building.

- Optimizing General Surfaces. The KG for correlated beliefs presented in this article requires that the covariance matrix $\Sigma^{0}$ be known. In Mes et al. [39], the $\mathrm{KG}$ concept is adapted to a problem for optimizing general functions, which may be nonconcave and which can be a function of discrete or categorical variables. The method uses statistical representation where the function is approximated using a weighted sum of estimates at different levels of aggregation. The KG is proven to find the optimum of the function in the limit, and appears to show good empirical convergence.

The KG has other useful theoretical properties. Frazier and Powell [9] shows that the $\mathrm{KG}$ policy is optimal (for any measurement budget) if there are only two choices, and that, for off-line problems, the policy is asymptotically optimal, which means that in the limit it will find the best alternative. Of course, many heuristic policies are asymptotically optimal (again, this applies only to off-line problems), but the KG algorithm is the only stationary policy that is both myopically optimal 
(which KG is by construction) and asymptotically optimal. This may be a reason why it appears to offer good empirical performance for intermediate measurement budgets.

The KG does not always work. It might consistently work well if the value of information is concave in the measurement budget, but this is not always the case. The marginal value of information may initially be quite small, when a single measurement is not enough to change a decision. But it may happen that, after some number of measurements, the marginal value starts to grow. This might arise, for example, if we are trying to find the best hitter for a baseball team. Watching a hitter for a few at bats teaches us almost nothing. It can take several hundred at bats before we can confidently say that one hitter is better than another. The value of information can then follow an S-curve, which means that it can be highly nonconvex for some applications.

The KG offers the type of general strategy for collecting information that steepest ascent has offered for a wide range of nonlinear maximization problems. It may not work best for all applications, but it may provide a valuable starting point for a wide range of problems.

\section{REFERENCES}

1. Bechhofer RE, Santner TJ, Goldsman DM. Design and analysis of experiments for statistical selection, screening, and multiple comparisons. New York: John Wiley \& Sons, Inc.; 1995.

2. Kim S-H, Nelson BL. Selecting the best system. Amsterdam: Elsevier; 2006. pp. 501-534, Chapter 17.

3. Goldsman DM, Nelson BL, Kim S-H. Statistical selection of the best system. Proceedings of the Winter Simulation Conference, 2005. Piscataway (NJ): IEEE Press; 2005. pp. 188-201. Available at http://ieeexplore .ieee.org/lpdocs/epic03/wrapper .htm?arnumber $=1574251$

4. Hastie T, Tibshirani R, Friedman J. The elements of statistical learning: data mining, inference, and prediction. New York: Springer; 2001.

5. Howard RA. Information value theory. IEEE Trans Syst Sci Cybern 1966;2(1):22-26.
Available at http://ieeexplore.ieee.org/xpls /abs_all.jsp?arnumber $=4082064$

6. Gupta S, Miescke K. Bayesian look ahead one-stage sampling allocations for selection of the best population. J Statistical Planning and Inference 1996;54(2):229-244. Available at http://linkinghub.elsevier.com/retrieve/pii/ 0378375895001697

7. Kushner HJ. A new method of locating the maximum point of an arbitrary multipeak curve in the presence of noise. J Basic Eng 1964;86:97-106.

8. Jones D, Schonlau M, Welch W. Efficient global optimization of expensive black-box functions. J Glob Optim 1998;13(4):455-492. Available at http://www.springerlink.com /index/M5878111M101017P.pdf

9. Frazier PI, Powell WB, Dayanik S. A knowledge gradient policy for sequential information collection. SIAM J Control and Optimization 2008;47(5):2410-2439.

10. Frazier PI, Powell WB, Dayanik S. The knowledge-gradient policy for correlated normal beliefs. INFORMS J. on Computing 2009;21(4):599-613. Available at http://joc .journal.informs.org/cgi/doi/10.1287/ijoc.1080 .0314

11. Chick SE, Branke J, Schmidt C. Sequential sampling to myopically maximize the expected value of information. INFORMS J. on Computing 2010;22(1):71-80. Available at http: // joc.journal.informs.org / cgi/doi/10.1287/ijoc .1090 .0327

12. Gittins J, Jones D. A dynamic allocation index for the sequential design of experiments. Amsterdam: North Holland; 1974. pp. 241-266.

13. Brezzi M, Lai T. Optimal learning and experimentation in bandit problems. J Econ Dyn Control 2002;27(1):87-108. Available at http://linkinghub.elsevier.com/retrieve/pii/ S0165188901000288

14. Chick SE, Gans N. Economic analysis of simulation selection problems. Manage Sci 2009;55(3):421-437.

15. Chen CH. An effective approach to smartly allocate computing budget for discrete event simulation. 34th IEEE Conference on Decision and Control, Volume 34, New Orleans (LA). pp. 2598-2603.

16. Yucesan E, Chen CH, Dai L, et al. A gradient approach for smartly allocating computing budget for discrete event simulation. In: Charnes J, Morrice D, Brunner D, 
et al., editors. Proceedings of the 1996 Winter Simulation Conference. Piscataway (NJ): IEEE Press; 1997. pp. 398-405.

17. Yucesan E, Chen $\mathrm{HC}$, Chen $\mathrm{CH}$, et al. New development of optimal computing budget allocation for discrete event simulation. Proceedings of the Winter Simulation Conference; Atlanta (GA): 1997. pp. 334-341.

18. Chick SE, Chen CH, Lin J, et al. Simulation budget allocation for further enhancing the efficiency of ordinal optimization. Discrete Event Dyn Syst 2000;10:251-270.

19. Chen HC, Chen CH, Yucesan E. Computing efforts allocation for ordinal optimization and discrete event simulation. IEEE Trans Automat Control 2000;45(5):960-964.

20. Chick SE, Inoue K. New two-stage and sequential procedures for selecting the best simulated system. Oper Res 2001;49(5):732-743. Available at http://www. jstor.org/stable/3088571

21. He D, Chick SE, Chen C-H. Opportunity cost and OCBA selection procedures in ordinal optimization for a fixed number of alternative systems. IEEE Trans Syst Man Cybern Part C Appl Rev 2007;37(5):951-961.

22. Fu MC, Hu J-Q, Chen C-H, et al. Simulation allocation for determining the best design in the presence of correlated sampling. INFORMS J Comput 2007;19:101-111.

23. Spall JC. Introduction to stochastic search and optimization: estimation, simulation and control. Hoboken (NJ): John Wiley \& Sons, Inc.; 2003.

24. Ryzhov IO, Powell WB, Frazier PI. The knowledge gradient algorithm for a general class of online learning problems; 2009. Accessed 2009. Available at http://www.castlelab. princeton.edu/Papers/RyzhovPowellFrazierOnlineKnowledgeGradientSept112009.pdf

25. Radner R, Stiglitz J. Volume 5, A nonconcavity in the value of information. Amsterdam: North-Holland Publishing Co.; 1984. chapter 3. pp. 33-52. Available at http://pages .stern.nyu.edu / rradner/publishedpapers/50 Nonconcavity.pdf

26. Chade H, Schlee EE. Another look at the radner-stiglitz nonconcavity in the value of information. J Econ Theory 2002;107(2): 421-452. Available at http://linkinghub. elsevier.com/retrieve/pii/S0022053101929606

27. Delara M, Gilotte L. A tight sufficient condition for Radner-Stiglitz nonconcavity in the value of information. J Econ Theory 2007;
137(1):696-708. Available at http:// linkinghub.elsevier.com/retrieve/pii/S002205 3107000373

28. Frazier PI, Powell WB. Paradoxes in learning: the marginal value of information and the problem of too many choices; 2010. Accessed 2010. Available at http://www.castlelab. princeton.edu/Papers/FrazierScurve-Jan19 2010.pdf

29. Berry DA, Fristedt B. Bandit problems. London: Chapman and Hall; 1985.

30. Pandey S, Chakrabarti D, Agarwal D. Multi-armed bandit problems with dependent arms. Proceedings of the 24th International Conference on Machine Learning. Corvallis (OR): ACM; 2007. pp. 721-728. Available at http://portal.acm.org/citation. cfm?id=1273496.1273587

31. Presman EL, Sonin IN. Sequential control with incomplete information: the Bayesian approach to multi-armed bandit problems. New York: Academic Press; 1990.

32. Mersereau A, Rusmevichientong P, Tsitsiklis J. A structured multiarmed bandit problem and the greedy policy. In: Medova-Dempster EA, Dempster MAH, editors. 47th IEEE Conference on Decision and Control. Piscataway (NJ): IEEE Press; CDC 2008. pp. 4945-4950.

33. Ginebra J, Clayton MK. Response surface bandits. J R Stat Soc Ser B (Methodological) 1995;57:771-784.

34. Agrawal R. The continuum-armed bandit problem. SIAM J Control Optim 1995;33(6):19-26. Available at http://link.aip. org/link/?SJCODC/33/1926/1

35. Kleinberg R. Nearly tight bounds for the continuum-armed bandit problem. In: Saul L, Weiss Y, Bottou L, editors. Advances in neural information processing systems. Cambridge (MA): MIT Press; 2004. pp. 697-704.

36. Ryzhov IO, Powell WB. Information collection on a graph. Oper Res 2010:1-35. Available at http://www.castlelab.princeton.edu/Papers/ RyzhovPowell-GraphPaperFeb102010.pdf

37. Negoescu DM, Frazier PI, Powell WB. The knowledge-gradient algorithm for sequencing experiments in drug discovery; 2010. Available at http://www.castlelab.princeton.edu/ Papers/Negoescu-OptimalLearningDrug Discovery_August242009.pdf

38. Ryzhov IO, Powell WB. A Monte-Carlo knowledge gradient method for learning abatement potential of emissions reduction technologies. 
In: Rossetti MD, Hill RR, Johansson B, et al., editors. Proceedings of the 2009 Winter Simulation Conference. Piscataway (NJ): IEEE Press; 2009.
39. Mes MRK, Powell WB, Frazier PI. Hierarchical knowledge gradient for sequential sampling; 2009. 